\title{
FDM for fractional parabolic equations with the Neumann condition
}

\author{
Allaberen Ashyralyev ${ }^{1,2}$ and Zafer Cakir ${ }^{3 *}$
}

${ }^{\text {*Correspondence: }}$

zafer@gumushane.edu.tr

${ }^{3}$ Department of Mathematical Engineering, Gumushane University,

Gumushane, Turkey

Full list of author information is

available at the end of the article

\begin{abstract}
In the present study, the first and second order of accuracy stable difference schemes for the numerical solution of the initial boundary value problem for the fractional parabolic equation with the Neumann boundary condition are presented. Almost coercive stability estimates for the solution of these difference schemes are obtained. The method is illustrated by numerical examples.
\end{abstract}

MSC: 34K37; 35R11; 35B35; 39A 14; 47B48

Keywords: fractional parabolic equations; Neumann condition; difference schemes; stability

\section{Introduction}

Mathematical modeling of fluid mechanics (dynamics, elasticity) and other areas of physics lead to fractional partial differential equations. Numerical methods and theory of solutions of the problems for fractional differential equations have been studied extensively by many researchers (see, e.g., [1-31] and the references given therein).

The method of operators as a tool for investigation of the well-posedness of boundary value problems for parabolic partial differential equations is well known (see, e.g., [32-41]). In paper [42], the initial value problem

$$
\frac{d u(t)}{d t}+D_{t}^{\frac{1}{2}} u(t)+A u(t)=f(t), \quad 0<t<T, \quad u(0)=0
$$

for the fractional differential equation in a Banach space $E$ with the strongly positive operator $A$ was investigated. This fractional differential equation corresponds to the Basset problem [43]. It represents a classical problem in fluid dynamics where the unsteady motion of a particle accelerates in a viscous fluid due to the gravity of force. Here $D_{t}^{\frac{1}{2}}=D_{0_{+}}^{\frac{1}{2}}$ is the standard Riemann-Liouville's derivative of order $\frac{1}{2}$.

The well-posedness of (1.1) in spaces of smooth functions was established. The coercive stability estimates for the solution of the $2 m$ th order multidimensional fractional parabolic equation and the one-dimensional fractional parabolic equation with nonlocal boundary conditions in space variable were obtained.

In paper [44], the stable first order of accuracy difference scheme for the approximate solution of initial value problem (1.1)

$$
\left\{\begin{array}{l}
\tau^{-1}\left(u_{k}-u_{k-1}\right)+A u_{k}+D_{\tau}^{\frac{1}{2}} u_{k}=f_{k}, \\
f_{k}=f\left(t_{k}\right), \quad t_{k}=k \tau, 1 \leq k \leq N, N \tau=T, \quad u_{0}=0
\end{array}\right.
$$

O 2013 Ashyralyev and Cakir; licensee Springer. This is an Open Access article distributed under the terms of the Creative Commons Attribution License (http://creativecommons.org/licenses/by/2.0), which permits unrestricted use, distribution, and reproduction in any medium, provided the original work is properly cited. 
was presented. Here (see, [45]),

$$
\begin{aligned}
& D_{\tau}^{\frac{1}{2}} u_{k}=\frac{1}{\sqrt{\pi}} \sum_{m=1}^{k} \frac{\Gamma\left(k-m+\frac{1}{2}\right)}{(k-m) !} \frac{u_{m}-u_{m-1}}{\tau^{\frac{1}{2}}}, \\
& \Gamma\left(k-m+\frac{1}{2}\right)=\int_{0}^{\infty} t^{k-m-\frac{1}{2}} e^{-t} d t .
\end{aligned}
$$

Let $F_{\tau}(E)$ be the linear space of mesh functions $\varphi^{\tau}=\left\{\varphi_{k}\right\}_{1}^{N}$ with values in the Banach space $E$. Next, on $F_{\tau}(E)$ we introduce the Banach space $C_{\tau}(E)=C\left([0, T]_{\tau}, E\right)$ with the norm

$$
\left\|\varphi^{\tau}\right\|_{C_{\tau}(E)}=\max _{1 \leq k \leq N}\left\|\varphi_{k}\right\|_{E}
$$

The well-posedness of (1.2) in difference analogues of spaces of smooth functions was established. Namely, we have the following theorems.

Theorem 1.1 Let $A$ be a strongly positive operator in a Banach space E. Then, for the solution $u^{\tau}=\left\{u_{k}\right\}_{1}^{N}$ in $C_{\tau}(E)$ of initial value problem (1.2) the stability inequality holds:

$$
\left\|\left\{D_{\tau}^{\frac{1}{2}} u_{k}\right\}_{1}^{N}\right\|_{C_{\tau}(E)}+\left\|\left\{\tau^{-1}\left(u_{k}-u_{k-1}\right)+A u_{k}\right\}_{1}^{N}\right\|_{C_{\tau}(E)} \leq M\left\|f^{\tau}\right\|_{C_{\tau}(E)} .
$$

Theorem 1.2 Let $A$ be a strongly positive operator in a Banach space E. Then, for the solution $u^{\tau}=\left\{u_{k}\right\}_{1}^{N}$ in $C_{\tau}(E)$ of initial value problem (1.2) the almost coercive stability inequality is valid:

$$
\begin{aligned}
& \left\|\left\{\tau^{-1}\left(u_{k}-u_{k-1}\right)\right\}_{1}^{N}\right\|_{C_{\tau}(E)}+\left\|\left\{A u_{k}\right\}_{1}^{N}\right\|_{C_{\tau}(E)} \\
& \leq M \min \left\{\ln \frac{1}{\tau}, 1+\ln \|A\|_{E \rightarrow E}\right\}\left\|f^{\tau}\right\|_{C_{\tau}(E)} .
\end{aligned}
$$

Here, and in future, positive constants, which can differ in time (hence: not a subject of precision) will be indicated with an $M$. On the other hand $M(\alpha, \beta, \ldots)$ is used to focus on the fact that the constant depends only on $\alpha, \beta, \ldots$.

Finally, the coercive stability and almost coercive stability estimates for the solution of difference schemes the first order of approximation in $t$ for the $2 m$ th order multidimensional fractional parabolic equation and the one-dimensional fractional parabolic equation with nonlocal boundary conditions in space variable were obtained.

In the present paper, applying the second order of approximation formula

$$
D_{\tau}^{1 / 2} u_{k}=\left\{\begin{array}{rlrl}
-d \sqrt{2} / 3 u_{0}+d \sqrt{2} / 3 u_{1}, & k=1, \\
2 d \sqrt{6} / 5 u_{0}+d \sqrt{6} / 5 u_{1}+d \sqrt{6} / 5 u_{2}, & k=2, \\
d \sum_{m=2}^{k-1}\left\{[(k-m) \lambda(k-m)+\mu(k-m)] u_{m-2}\right. & \\
& +[(2 m-2 k-1) \lambda(k-m)-2 \mu(k-m)] u_{m-1} & \\
& \left.+[(k-m+1) \lambda(k-m)+\mu(k-m)] u_{m}\right\} & \\
& +\frac{d}{6 \sqrt{2}}\left[-u_{k-2}-4 u_{k-1}+5 u_{k}\right], & 3 \leq k \leq N
\end{array}\right.
$$

for

$$
D_{t}^{1 / 2} u\left(t_{k}-\tau / 2\right)=\frac{1}{\Gamma(1 / 2)} \int_{0}^{t_{k}-\tau / 2}\left(t_{k}-\tau / 2-s\right)^{-1 / 2} u^{\prime}(s) d s,
$$


and using the Crank-Nicholson difference scheme for parabolic equations, we present the second order of accuracy difference scheme

$$
\left\{\begin{array}{l}
\tau^{-1}\left(u_{k}-u_{k-1}\right)+\frac{1}{2} A\left(u_{k}+u_{k-1}\right)+D_{\tau}^{\frac{1}{2}} u_{k}=f_{k}, \\
f_{k}=f\left(t_{k}-\frac{\tau}{2}\right), \quad t_{k}=k \tau, 1 \leq k \leq N, N \tau=T, \quad u_{0}=0
\end{array}\right.
$$

for the approximate solution of initial value problem (1.1). Here,

$$
\begin{aligned}
& d=\frac{2}{\sqrt{\pi \tau}}, \quad \lambda(r)=\sqrt{r+1 / 2}-\sqrt{r-1 / 2}, \\
& \mu(r)=-\frac{1}{3}\left((r+1 / 2)^{3 / 2}-(r-1 / 2)^{3 / 2}\right) .
\end{aligned}
$$

The well-posedness of (1.7) in $C_{\tau}(E)$ is established. In applications, the initial boundary value problem for the fractional parabolic equation

$$
\left\{\begin{array}{l}
\frac{\partial u(t, x)}{\partial t}+D_{t}^{1 / 2} u(t, x)-\sum_{p=1}^{m} a_{p}(x) \frac{\partial^{2} u(t, x)}{\partial x_{p}^{2}}+\sum_{p=1}^{m} b_{p}(x) \frac{\partial u(t, x)}{\partial x_{p}}+\sigma u(t, x)=f(t, x) \\
\quad x=\left(x_{1}, \ldots, x_{m}\right) \in \Omega, 0<t<T \\
\frac{\partial u(t, x)}{\partial \bar{n}}=0, \quad x \in S, 0 \leq t \leq T \\
u(0, x)=0, \quad x \in \bar{\Omega}
\end{array}\right.
$$

is considered. Here, $\Omega$ is the open cube in the $m$-dimensional Euclidean space

$$
\mathbb{R}^{m}:\left\{x \in \Omega: x=\left(x_{1}, \ldots, x_{m}\right) ; 0<x_{j}<1,1 \leq j \leq m\right\}
$$

with boundary $S, \bar{\Omega}=\Omega \cup S, a_{p}(x)$ and $b_{p}(x)(x \in \Omega)$ and $f(t, x)(t \in(0, T), x \in \Omega)$ are given smooth functions and $a_{p}(x) \geq a>0, \sigma>0$ and $\vec{n}$ is the normal vector to $S$.

The first and second order of accuracy difference schemes for the approximate solution of problem (1.8) are presented. The almost coercive stability estimates for the solution of these difference schemes are established. The theoretical statements for the solution of these difference schemes for one-dimensional fractional parabolic equations are supported by numerical examples.

\section{The well-posedness of difference scheme}

It is clear that the following representation formula

$$
u_{k}=-\sum_{s=1}^{k} B^{k-s} C D_{\tau}^{\frac{1}{2}} u_{s} \tau+\sum_{s=1}^{k} B^{k-s} C f_{s} \tau, \quad 1 \leq k \leq N
$$

holds for the solution of problem (1.7). Here, $C=\left(I+\frac{\tau A}{2}\right)^{-1}$ and $B=\left(I-\frac{\tau A}{2}\right) C$.

Theorem 2.1 Let $A$ be a strongly positive operator in a Banach space E. Then, for the solution $u^{\tau}=\left\{u_{k}\right\}_{1}^{N}$ in $C_{\tau}(E)$ of initial value problem (1.2) the following stability inequality holds:

$$
\left\|\left\{D_{\tau}^{\frac{1}{2}} u_{k}\right\}_{1}^{N}\right\|_{C_{\tau}(E)}+\left\|\left\{\tau^{-1}\left(u_{k}-u_{k-1}\right)+\frac{A}{2}\left(u_{k}+u_{k-1}\right)\right\}_{1}^{N}\right\|_{C_{\tau}(E)} \leq M\left\|f^{\tau}\right\|_{C_{\tau}(E)} .
$$


Proof Using formulas (2.1) and (1.6), we get

$$
\begin{aligned}
D_{\tau}^{\frac{1}{2}} u_{1}= & d \sqrt{2} / 3\left\{-C D_{\tau}^{\frac{1}{2}} u_{1} \tau+C f_{1} \tau\right\} \\
D_{\tau}^{\frac{1}{2}} u_{2}= & d \sqrt{6} / 5\left\{-C^{2} D_{\tau}^{\frac{1}{2}} u_{1} \tau-\frac{C}{2} D_{\tau}^{\frac{1}{2}} u_{2} \tau+C^{2} f_{1} \tau+\frac{C}{2} f_{2} \tau\right\} \\
D_{\tau}^{\frac{1}{2}} u_{k}= & d \sum_{m=2}^{k-1}\{[(k-m) \lambda(k-m)+\mu(k-m)] \\
& \times\left[-\sum_{s=1}^{m-2} B^{m-2-s} C D_{\tau}^{\frac{1}{2}} u_{s} \tau+\sum_{s=1}^{m-2} B^{m-2-s} C f_{s} \tau\right] \\
& +[(2 m-2 k-1) \lambda(k-m)-2 \mu(k-m)] \\
& \times\left[-\sum_{s=1}^{m-1} B^{m-1-s} C D_{\tau}^{\frac{1}{2}} u_{s} \tau+\sum_{s=1}^{m-1} B^{m-1-s} C f_{s} \tau\right] \\
& +[(k-m+1) \lambda(k-m)+\mu(k-m)] \\
& \left.\times\left[-\sum_{s=1}^{m} B^{m-s} C D_{\tau}^{\frac{1}{2}} u_{s} \tau+\sum_{s=1}^{m} B^{m-s} C f_{s} \tau\right]\right\} \\
& +\frac{d}{6 \sqrt{2}}\left\{-\left[-\sum_{s=1}^{k-2} B^{k-2-s} C D_{\tau}^{\frac{1}{2}} u_{s} \tau+\sum_{s=1}^{k-2} B^{k-2-s} C f_{s} \tau\right]\right. \\
& -4\left[-\sum_{s=1}^{k-1} B^{k-1-s} C D_{\tau}^{\frac{1}{2}} u_{s} \tau+\sum_{s=1}^{k-1} B^{k-1-s} C f_{s} \tau\right] \\
& {\left.\left[-\sum_{s=1}^{k} B^{k-s} C D_{\tau}^{\frac{1}{2}} u_{s} \tau+\sum_{s=1}^{k} B^{k-s} C f_{s} \tau\right]\right\}, 3 \leq k \leq N . }
\end{aligned}
$$

Now, let us first estimate $z_{k}=\left\|D_{\tau}^{\frac{1}{2}} u_{k}\right\|_{E}$ for any $1 \leq k \leq N$. Using formula (2.3) and the estimate

$$
\|C\|_{E \rightarrow E} \leq M
$$

we get

$$
\begin{aligned}
z_{1} & \leq\|C\|_{E \rightarrow E}\left[\left\|D_{\tau}^{\frac{1}{2}} u_{1}\right\|_{E}+\left\|f_{1}\right\|_{E}\right] \sqrt{\tau} \leq M \sqrt{\tau}\left[z_{1}+\left\|f_{1}\right\|_{E}\right], \\
z_{2} & \leq\left\{\frac{1}{2}\|C\|_{E \rightarrow E}\left[\left\|D_{\tau}^{\frac{1}{2}} u_{2}\right\|_{E}+\left\|f_{2}\right\|_{E}\right]+\|C\|_{E \rightarrow E}^{2}\left[\left\|D_{\tau}^{\frac{1}{2}} u_{1}\right\|_{E}+\left\|f_{1}\right\|_{E}\right]\right\} \sqrt{\tau} \\
& \leq M \sqrt{\tau}\left[z_{1}+z_{2}+\left\|f_{1}\right\|_{E}+\left\|f_{2}\right\|_{E}\right] .
\end{aligned}
$$

Now we consider the case $3 \leq k \leq N$. Applying formula (2.3), the triangle inequality and estimates [46]

$$
\left\|A B^{k} C^{2}\right\|_{E \rightarrow E} \leq \frac{M}{k \tau}, \quad\left\|B^{k} C\right\|_{E \rightarrow E} \leq M, \quad 1 \leq k \leq N,
$$


we get

$$
z_{k} \leq M_{3} \sum_{s=1}^{k-1} \frac{1}{\sqrt{(k-s) \tau}} \tau\left(z_{s}+\left\|f_{s}\right\|_{E}\right)+M_{4}\left(z_{k}+\left\|f_{k}\right\|_{E}\right) \tau^{\frac{1}{2}}
$$

Applying the difference analogue of the integral inequality and inequalities (2.5), (2.6) and (2.8), we get

$$
\left\|\left\{D_{\tau}^{\frac{1}{2}} u_{k}\right\}_{1}^{N}\right\|_{C_{\tau}(E)}=\left\|\left\{z_{k}\right\}_{1}^{N}\right\|_{C_{\tau}(E)} \leq M\left\|f^{\tau}\right\|_{C_{\tau}(E)} .
$$

Using the triangle inequality and equation (1.2), we get

$$
\begin{aligned}
\left\|\left\{\tau^{-1}\left(u_{k}-u_{k-1}\right)+\frac{A}{2}\left(u_{k}+u_{k-1}\right)\right\}_{1}^{N}\right\|_{C_{\tau}(E)} & \leq\left[\left\|f^{\tau}\right\|_{C_{\tau}(E)}+\left\|\left\{D_{\tau}^{\frac{1}{2}} u_{k}\right\}_{1}^{N}\right\|_{C_{\tau}(E)}\right] \\
& \leq M_{1}\left\|f^{\tau}\right\|_{C_{\tau}(E)} .
\end{aligned}
$$

Estimate (2.2) follows from estimates (2.9) and (2.10). Theorem 2.1 is proved.

Theorem 2.2 Let $A$ be a strongly positive operator in a Banach space E. Then, for the solution $u^{\tau}=\left\{u_{k}\right\}_{1}^{N}$ in $C_{\tau}(E)$ of initial value problem (1.2) the almost coercive stability inequality is valid:

$$
\begin{gathered}
\left\|\left\{\tau^{-1}\left(u_{k}-u_{k-1}\right)\right\}_{1}^{N}\right\|_{C_{\tau}(E)}+\left\|\left\{\frac{A}{2}\left(u_{k}+u_{k-1}\right)\right\}_{1}^{N}\right\|_{C_{\tau}(E)} \\
\leq M \min \left\{\ln \frac{1}{\tau}, 1+\ln \|A\|_{E \rightarrow E}\right\}\left\|f^{\tau}\right\|_{C_{\tau}(E)} .
\end{gathered}
$$

Proof Using formula (2.1), we get

$$
\begin{aligned}
& \tau^{-1}\left(u_{k}-u_{k-1}\right) \\
& \quad=-\frac{C}{2} D_{\tau}^{\frac{1}{2}} u_{k}-\sum_{s=1}^{k-1} A B^{k-1-s} C^{2} D_{\tau}^{\frac{1}{2}} u_{s} \tau+\frac{C}{2} f_{k}+\sum_{s=1}^{k-1} A B^{k-1-s} C^{2} f_{s} \tau .
\end{aligned}
$$

The proof of estimate

$$
\left\|\left\{\tau^{-1}\left(u_{k}-u_{k-1}\right)\right\}_{1}^{N}\right\|_{C_{\tau}(E)} \leq M \min \left\{\ln \frac{1}{\tau}, 1+\ln \|A\|_{E \rightarrow E}\right\}\left\|f^{\tau}\right\|_{C_{\tau}(E)}
$$

for the solution of initial value problem (1.2) is based on formula (2.12) and estimate (2.2) and the following estimates [46]:

$$
\begin{aligned}
& \max _{1 \leq k \leq N}\left\|\frac{C}{2} f_{k}+\sum_{s=1}^{k-1} A B^{k-1-s} C^{2} f_{s} \tau\right\|_{E} \\
& \leq M \min \left\{\ln \frac{1}{\tau}, 1+\ln \|A\|_{E \rightarrow E}\right\}\left\|f^{\tau}\right\|_{C(E)},
\end{aligned}
$$




$$
\begin{aligned}
& \max _{1 \leq k \leq N}\left\|\frac{C}{2} f_{k}+\sum_{s=1}^{k-1} A B^{k-1-s} C^{2} D_{\tau}^{\frac{1}{2}} u_{s} \tau\right\|_{E} \\
& \leq M \min \left\{\ln \frac{1}{\tau}, 1+\ln \|A\|_{E \rightarrow E}\right\}\left\|\left\{D_{\tau}^{\frac{1}{2}} u_{k}\right\}_{1}^{N}\right\|_{C(E)} .
\end{aligned}
$$

Using these estimates, the triangle inequality and equation (1.2), we get

$$
\left\|\left\{\frac{A}{2}\left(u_{k}+u_{k-1}\right)\right\}_{1}^{N}\right\|\left\|_{C_{\tau}(E)} \leq M_{1} \min \left\{\ln \frac{1}{\tau}, 1+\ln \|A\|_{E \rightarrow E}\right\}\right\| f^{\tau} \|_{C(E)} .
$$

Estimate (2.11) follows from estimates (2.13) and (2.14). Theorem 2.2 is proved.

\section{Applications}

Now, we consider the applications of Theorems 2.1 and 2.2 to initial boundary value problem (1.8). The discretization of problem (1.8) is carried out in two steps. In the first step, let us define the grid space

$$
\left\{\begin{aligned}
\bar{\Omega}_{h}= & \left\{x=x_{p}=\left(h_{1} p_{1}, \ldots, h_{m} p_{m}\right), p=\left(p_{1}, \ldots, p_{m}\right)\right. \\
& \left.0 \leq p_{j} \leq M_{j}, h_{j} M_{j}=1, j=1, \ldots, m\right\} \\
\Omega_{h}= & \bar{\Omega}_{h} \cap \Omega, \quad S_{h}=\bar{\Omega}_{h} \cap S .
\end{aligned}\right.
$$

We introduce the Banach space $C_{h}=C\left(\bar{\Omega}_{h}\right)$ of the grid function $\varphi^{h}(x)=\left\{\varphi\left(h_{1} p_{1}, \ldots\right.\right.$, $\left.h_{m} p_{m}\right)$ \} defined on $\bar{\Omega}$, equipped with the norm

$$
\left\|\varphi^{h}\right\|_{C\left(\bar{\Omega}_{h}\right)}=\max _{x \in \bar{\Omega}_{h}}\left|\varphi^{h}(x)\right| .
$$

To the differential operator $A^{x}$ generated by problem (1.8), we assign the difference operator $A_{h}^{x}$ by the formula

$$
A_{h}^{x} u^{h}(x)=-\sum_{p=1}^{m} a_{p}(x) u_{\bar{x} x_{p}, j_{p}}^{h}(x)+\sum_{p=1}^{m} b_{p}(x) u_{x_{x, j_{p}}^{h}}^{h}(x)+\sigma u^{h}(x)
$$

acting in the space of grid functions $u^{h}(x)$, satisfying the conditions $D^{h} u^{h}(x)=0$ for all $x \in S_{h}$. Here, $D^{h} u^{h}(x)$ is the first or second order of approximation of $\frac{\partial u}{\partial \breve{n}}$. It is known that (see, $[47,48]) A_{h}^{x}$ is a strongly positive definite operator in $C\left(\bar{\Omega}_{h}\right)$. With the help of $A_{h}^{x}$ we arrive at the initial boundary value problem

$$
\left\{\begin{array}{l}
\frac{d v^{h}(t, x)}{d t}+D_{t}^{1 / 2} v^{h}(t, x)+A_{h}^{x} v^{h}(t, x)=f^{h}(t, x), \quad 0<t<T, x \in \Omega_{h}, \\
v^{h}(0, x)=0, \quad x \in \bar{\Omega}
\end{array}\right.
$$

for a finite system of ordinary fractional differential equations.

In the second step, applying the first order of approximation formula defined by (1.3) for

$$
D_{t}^{1 / 2} u\left(t_{k}\right)=\frac{1}{\Gamma(1 / 2)} \int_{0}^{t_{k}}\left(t_{k}-s\right)^{-1 / 2} u^{\prime}(s) d s
$$


and using the first order of accuracy stable difference scheme for parabolic equations, we can present the first order of accuracy difference scheme

$$
\left\{\begin{array}{l}
\frac{u_{k}^{h}(x)-u_{k-1}^{h}(x)}{\tau}+D_{\tau}^{1 / 2} u_{k}^{h}(x)+A_{h}^{x} u_{k}^{h}(x)=f_{k}^{h}(x), \quad x \in \bar{\Omega}_{h}, \\
f_{k}^{h}(x)=f^{h}\left(t_{k}, x\right), \quad t_{k}=k \tau, 1 \leq k \leq N, N \tau=T, \\
u_{0}^{h}(x)=0, \quad x \in \bar{\Omega}_{h}
\end{array}\right.
$$

for the approximate solution of problem (1.8).

Moreover, applying the second order of approximation formula defined by (1.6) for

$$
D_{t}^{1 / 2} u\left(t_{k}-\tau / 2\right)=\frac{1}{\Gamma(1 / 2)} \int_{0}^{t_{k}-\tau / 2}\left(t_{k}-\tau / 2-s\right)^{-1 / 2} u^{\prime}(s) d s
$$

and using the Crank-Nicholson difference scheme for parabolic equations, we can present the second order of accuracy difference scheme

$$
\left\{\begin{array}{l}
\frac{u_{k}^{h}(x)-u_{k-1}^{h}(x)}{\tau}+D_{\tau}^{1 / 2} u_{k}^{h}(x)+\frac{1}{2} A_{h}^{x}\left(u_{k}^{h}(x)+u_{k-1}^{h}(x)\right)=f_{k}^{h}(x), \quad x \in \bar{\Omega}_{h}, \\
f_{k}^{h}(x)=f\left(t_{k}-\frac{\tau}{2}, x\right), \quad t_{k}=k \tau, 1 \leq k \leq N, N \tau=T, \\
u_{0}^{h}(x)=0, \quad x \in \bar{\Omega}_{h}
\end{array}\right.
$$

for the approximate solution of problem (1.8).

Theorem 3.1 Let $\tau$ and $|h|=\sqrt{h_{1}^{2}+\cdots+h_{n}^{2}}$ be sufficiently small numbers. Then the solutions of difference scheme (3.2) satisfy the following almost coercive stability estimates:

$$
\begin{aligned}
& \max _{1 \leq k \leq N}\left\|\frac{u_{k}^{h}-u_{k-1}^{h}}{\tau}\right\|_{C_{h}} \leq M_{2} \ln \frac{1}{\tau+|h|} \max _{1 \leq k \leq N}\left\|f_{k}^{h}\right\|_{C_{h}}, \\
& \max _{1 \leq k \leq N} \sum_{p=1}^{m}\left\|\left(u_{k}^{h}\right)_{\bar{x}_{p} x_{p}, j_{p}}\right\|_{C_{h}} \leq M_{2}(\sigma) \ln \frac{1}{h} \ln \frac{1}{\tau+|h|} \max _{1 \leq k \leq N}\left\|f_{k}^{h}\right\|_{C_{h} .}
\end{aligned}
$$

The proof of Theorem 3.1 is based on the abstract Theorem 1.2 and on the estimate

$$
\min \left\{\ln \frac{1}{\tau}, 1+\left|\ln \left\|A_{h}^{x}\right\|_{C_{h} \rightarrow C_{h}}\right|\right\} \leq M(\sigma) \ln \frac{1}{\tau+|h|}
$$

as well as on the positivity of the operator $A_{h}^{x}$ in $C_{h}[47,48]$, along with the following theorem on the almost coercivity inequality for the solution of the elliptic difference equation in $C_{h}$.

Theorem 3.2 [49] Let $|h|=\sqrt{h_{1}^{2}+\cdots+h_{n}^{2}}$ be sufficiently small number. Then, for the solutions of the elliptic difference equation

$$
A_{h}^{x} u^{h}(x)=\omega^{h}(x), \quad x \in \Omega_{h}
$$

the following almost coercivity inequality

$$
\sum_{p=1}^{m}\left\|u_{\bar{x}_{p} x_{p}, j_{p}}^{h}\right\|_{C_{h}} \leq M(\sigma) \ln \frac{1}{|h|}\left\|\omega^{h}\right\|_{C_{h}}
$$

is valid. 
Theorem 3.3 Let $\tau$ and $|h|=\sqrt{h_{1}^{2}+\cdots+h_{m}^{2}}$ be sufficiently small numbers. Then the solutions of difference scheme (3.3) satisfy the following almost coercive stability estimates:

$$
\begin{aligned}
& \max _{1 \leq k \leq N}\left\|\frac{u_{k}^{h}-u_{k-1}^{h}}{\tau}\right\|_{C_{h}} \leq M_{3} \ln \frac{1}{\tau+|h|} \max _{1 \leq k \leq N}\left\|f_{k}^{h}\right\|_{C_{h}}, \\
& \max _{1 \leq k \leq N} \frac{1}{2} \sum_{p=1}^{m}\left\|\left(u_{k}^{h}+u_{k-1}^{h}\right)_{\bar{x}_{p} x_{p}, j_{p}}\right\| C_{C_{h}} \leq M_{3}(\sigma) \ln \frac{1}{h} \ln \frac{1}{\tau+|h|} \max _{1 \leq k \leq N}\left\|f_{k}^{h}\right\|_{C_{h}} .
\end{aligned}
$$

The proof of Theorem 3.3 is based on the abstract Theorem 2.2 and on estimate (3.4) and on the positivity of the operator $A_{h}^{x}$ in $C_{h}$ and on Theorem 3.2 on the almost coercivity inequality for the solution of the elliptic difference equation in $C_{h}$.

Note that one has not been able to get a sharp estimate for the constants figuring in the almost coercive stability estimates of Theorems 3.1 and 3.3. Therefore, our interest in the present paper is studying the difference schemes (3.2) and (3.3) by numerical experiments. Applying these difference schemes, the numerical methods are proposed in the following section for solving the one-dimensional fractional parabolic partial differential equation. The method is illustrated by numerical experiments.

\section{Numerical results}

For the numerical result, the initial value problem

$$
\left\{\begin{aligned}
& \frac{\partial u(t, x)}{\partial t}+ D_{t}^{1 / 2} u(t, x)-\frac{\partial}{\partial x}\left((1+x) \frac{\partial u(t, x)}{\partial x}\right)+u(t, x)=f(t, x) \\
& f(t, x)= {\left[3+t+\frac{16 \sqrt{t}}{5 \sqrt{\pi}}+(1+x) \pi^{2} t\right] t^{2} \cos \pi x } \\
&+\pi t^{3} \sin \pi x, \quad 0<t<1,0<x<1, \\
& u_{x}(t, 0)= u_{x}(t, 1)=0, \quad 0 \leq t \leq 1 \\
& u(0, x)=0, \quad 0 \leq x \leq 1
\end{aligned}\right.
$$

for the one-dimensional fractional parabolic partial differential equation is considered. The exact solution of problem (4.1) is $u(t, x)=t^{3} \cos \pi x$.

\subsection{First order of accuracy difference scheme}

Applying difference scheme (3.2), we obtain

$$
\left\{\begin{aligned}
& \frac{u_{n}^{k}-u_{n}^{k-1}}{\tau}+\frac{1}{\sqrt{\pi}} \sum_{r=1}^{k} \frac{\Gamma(k-r+1 / 2)}{(k-r) !}\left(\frac{u_{n}^{r}-u_{n}^{r-1}}{\tau^{1 / 2}}\right) \\
& \quad-\frac{1}{h}\left[\left(1+x_{n+1}\right) \frac{u_{n+1}^{k}-u_{n}^{k}}{h}-\left(1+x_{n}\right) \frac{u_{n}^{k}-u_{n-1}^{k}}{h}\right]+u_{n}^{k}=\varphi_{n}^{k}, \\
& \varphi_{n}^{k}=f\left(t_{k}, x_{n}\right), \quad t_{k}=k \tau, 1 \leq k \leq N, x_{n}=n h, 1 \leq n \leq M-1, \\
& u_{0}^{k}=u_{1}^{k}, \quad u_{M-1}^{k}=u_{M}^{k}, \quad 0 \leq k \leq N, \\
& u_{n}^{0}=0, \quad 0 \leq n \leq M .
\end{aligned}\right.
$$

It can be rewritten in the matrix form

$$
\left\{\begin{array}{l}
A U_{n+1}+B U_{n}+C U_{n-1}=D \varphi_{n}, \quad 1 \leq n \leq M-1, \\
U_{0}=U_{1}, \quad U_{M-1}=U_{M},
\end{array}\right.
$$


where

$$
\begin{aligned}
& A=\left[\begin{array}{cccccc}
0 & 0 & 0 & \cdots & 0 & 0 \\
0 & a_{n} & 0 & \cdots & 0 & 0 \\
0 & 0 & a_{n} & \cdots & 0 & 0 \\
\cdots & \cdots & \cdots & \cdots & \cdots & \cdots \\
0 & 0 & 0 & \cdots & a_{n} & 0 \\
0 & 0 & 0 & \cdots & 0 & a_{n}
\end{array}\right]_{(N+1) \times(N+1)} \\
& B=\left[\begin{array}{cccccc}
b_{11} & 0 & 0 & \cdots & 0 & 0 \\
b_{21} & b_{22} & 0 & \cdots & 0 & 0 \\
b_{31} & b_{32} & b_{33} & \cdots & 0 & 0 \\
\cdots & \cdots & \cdots & \cdots & \cdots & \cdots \\
b_{N 1} & b_{N 2} & b_{N 3} & \cdots & b_{N N} & 0 \\
b_{N+1,1} & b_{N+1,2} & b_{N+1,3} & \cdots & b_{N+1, N} & b_{N+1, N+1}
\end{array}\right]_{(N+1) \times(N+1)} \\
& C=\left[\begin{array}{cccccc}
0 & 0 & 0 & \cdots & 0 & 0 \\
0 & c_{n} & 0 & \cdots & 0 & 0 \\
0 & 0 & c_{n} & \cdots & 0 & 0 \\
\cdots & \cdots & \cdots & \cdots & \cdots & \cdots \\
0 & 0 & 0 & \cdots & c_{n} & 0 \\
0 & 0 & 0 & \cdots & 0 & c_{n}
\end{array}\right]_{(N+1) \times(N+1)} \\
& D=\left[\begin{array}{cccccc}
0 & 0 & 0 & \cdots & 0 & 0 \\
0 & 1 & 0 & \cdots & 0 & 0 \\
0 & 0 & 1 & \cdots & 0 & 0 \\
\cdots & \cdots & \cdots & \cdots & \cdots & \cdots \\
0 & 0 & 0 & \cdots & 1 & 0 \\
0 & 0 & 0 & \cdots & 0 & 1
\end{array}\right]_{(N+1) \times(N+1)} \\
& \varphi_{n}=\left[\begin{array}{c}
\varphi_{n}^{0} \\
\varphi_{n}^{1} \\
\varphi_{n}^{2} \\
\vdots \\
\varphi_{n}^{N-1} \\
\varphi_{n}^{N}
\end{array}\right]_{(N+1) \times 1}, \quad U_{q}=\left[\begin{array}{c}
u_{q}^{0} \\
u_{q}^{1} \\
u_{q}^{2} \\
\vdots \\
u_{q}^{N-1} \\
u_{q}^{N}
\end{array}\right]_{(N+1) \times 1} \quad, \quad q=n \pm 1, n, \\
& a_{n}=-\frac{1+x_{n+1}}{h^{2}}, \quad c_{n}=-\frac{1+x_{n}}{h^{2}}, \quad b_{11}=1, \quad b_{21}=-\frac{1}{\sqrt{\tau}}-\frac{1}{\tau}, \\
& b_{22}=\frac{1}{\sqrt{\tau}}+\frac{1}{\tau}+\frac{2+x_{n+1}+x_{n}}{h^{2}}+1, \quad b_{31}=-\frac{\Gamma(1+1 / 2)}{\sqrt{\pi \tau}}, \\
& b_{32}=\frac{\Gamma(1+1 / 2)-\Gamma(1 / 2)}{\sqrt{\pi \tau}}-\frac{1}{\tau}, \quad b_{33}=\frac{1}{\sqrt{\tau}}+\frac{1}{\tau}+\frac{2+x_{n+1}+x_{n}}{h^{2}}+1 \text {, } \\
& b_{i j}= \begin{cases}-\frac{\Gamma(i-2+1 / 2)}{\sqrt{\pi \tau}(i-2) !}, & j=1, \\
\sqrt{\pi \tau}\left[\frac{\Gamma(i-j+1 / 2)}{(i-j) !}-\frac{\Gamma(i-j-1+1 / 2)}{(i-j-1) !}\right], & 2 \leq j \leq i-2, \\
\sqrt{\pi \tau}[\Gamma(1+1 / 2)-\Gamma(1 / 2)]-\frac{1}{\tau}, & j=i-1, \\
\frac{1}{\sqrt{\tau}}+\frac{1}{\tau}+\frac{2+x_{n+1}+x_{n}}{h^{2}}+1, & j=i, \\
0, & i<j \leq N+1\end{cases}
\end{aligned}
$$


for $i=4,5, \ldots, N+1$ and

$$
\varphi_{n}^{k}=\left[3+k \tau+\frac{16 \sqrt{k \tau}}{5 \sqrt{\pi}}+(1+n h) \pi^{2} k \tau\right](k \tau)^{2} \cos (\pi n h)+\pi(k \tau)^{3} \sin (\pi n h) .
$$

So, we have the second order difference equation with respect to $n$ matrix coefficients. This type system was developed by Samarskii and Nikolaev [50]. To solve this difference equation, we have applied a procedure for difference equation with respect to $k$ matrix coefficients. Hence, we seek a solution of the matrix equation in the following form:

$$
U_{j}=\alpha_{j+1} U_{j+1}+\beta_{j+1}, \quad U_{M}=\left(I-\alpha_{M}\right)^{-1} \beta_{M}, \quad j=M-1, \ldots, 2,1,
$$

where $\alpha_{j}(j=1,2, \ldots, M)$ are $(N+1) \times(N+1)$ square matrices and $\beta_{j}(j=1,2, \ldots, M)$ are $(N+1) \times 1$ column matrices defined by

$$
\begin{aligned}
& \alpha_{j+1}=-\left(B+C \alpha_{j}\right)^{-1} A, \\
& \beta_{j+1}=\left(B+C \alpha_{j}\right)^{-1}\left(D \varphi_{j}-C \beta_{j}\right), \quad j=1,2, \ldots, M-1,
\end{aligned}
$$

where $j=1,2, \ldots, M-1, \alpha_{1}$ is the $(N+1) \times(N+1)$ identity matrix and $\beta_{1}$ is the $(N+1) \times 1$ zero matrix.

\subsection{Second order of accuracy difference scheme}

Applying the formulas

$$
\begin{aligned}
& u_{x}\left(t_{k}, 0\right)=\frac{u_{1}^{k}-u_{0}^{k}}{h}-\frac{h}{2} u_{x x}\left(t_{k}, 0\right)+o\left(h^{2}\right), \quad 0 \leq k \leq N, \\
& u_{x}\left(t_{k}, M\right)=\frac{3 u_{M}^{k}-4 u_{M-1}^{k}+u_{M-2}^{k}}{2 h}+o\left(h^{2}\right), \quad 0 \leq k \leq N, \\
& u_{t}\left(t_{k}, 0\right)=\frac{u_{0}^{k+1}-u_{0}^{k-1}}{2 \tau}+o\left(\tau^{2}\right), \quad 1 \leq k \leq N-1, \\
& u_{t}\left(t_{N}, 0\right)=\frac{3 u_{0}^{N}-4 u_{0}^{N-1}+u_{0}^{N-2}}{2 \tau}+o\left(\tau^{2}\right), \quad k=N
\end{aligned}
$$

and using difference scheme (3.3), we obtain the second order of accuracy difference scheme in $t$ and in $x$

$$
\left\{\begin{array}{l}
\frac{u_{n}^{k}-u_{n}^{k-1}}{\tau}+D_{\tau}^{1 / 2} u_{n}^{k}-\frac{1}{2}\left[\left(1+x_{n}\right) \frac{u_{n+1}^{k}-2 u_{n}^{k}+u_{n-1}^{k}}{h^{2}}+\frac{u_{n+1}^{k}-u_{n-1}^{k}}{2 h}-u_{n}^{k}\right. \\
\left.\quad+\left(1+x_{n}\right) \frac{u_{n+1}^{k-1}-2 u_{n}^{k-1}+u_{n-1}^{k-1}}{h^{2}}+\frac{u_{n+1}^{k-1}-u_{n-1}^{k-1}}{2 h}-u_{n}^{k-1}\right]=\varphi_{n}^{k}, \\
\varphi_{n}^{k}=f\left(t_{k}-\tau / 2, x_{n}\right), \quad t_{k}=k \tau, x_{n}=n h, 1 \leq k \leq N, 1 \leq n \leq M-1, \\
u_{0}^{0}=0, \quad k=0, \\
-\frac{h}{4 \tau} u_{0}^{k-1}+\left[\frac{1}{h}+\frac{h}{2} D_{\tau}^{1 / 2}+\frac{h}{2}\right] u_{0}^{k}+\frac{h}{4 \tau} u_{0}^{k+1}=\frac{1}{h} u_{1}^{k}+\frac{h}{2} \varphi_{0}^{k}, \quad 1 \leq k \leq N-1, \\
\frac{h}{4 \tau} u_{0}^{N-2}-\frac{h}{\tau} u_{0}^{N-1}+\left[\frac{1}{h}+\frac{3 h}{4 \tau}+\frac{h}{2} D_{\tau}^{1 / 2}+\frac{h}{2}\right] u_{0}^{N}=\frac{1}{h} u_{1}^{N}+\frac{h}{2} \varphi_{0}^{N}, \quad k=N, \\
3 u_{M}^{k}-4 u_{M-1}^{k}+u_{M-2}^{k}=0, \quad 0 \leq k \leq N, \\
u_{n}^{0}=0, \quad 0 \leq n \leq M .
\end{array}\right.
$$


Here, $D_{\tau}^{1 / 2} u_{n}^{k}$ is the fractional difference derivative defined by the formula (1.6). It can be rewritten in the matrix form

$$
\left\{\begin{array}{l}
A U_{n+1}+B U_{n}+C U_{n-1}=D \varphi_{n}, \quad 1 \leq n \leq M-1, \\
E U_{0}=F U_{1}+R \varphi_{0}, \quad 3 U_{M}-4 U_{M-1}+U_{M-2}=0,
\end{array}\right.
$$

where

$$
\begin{aligned}
& A=\left[\begin{array}{cccccc}
0 & 0 & 0 & \cdots & 0 & 0 \\
a_{n} & a_{n} & 0 & \cdots & 0 & 0 \\
0 & a_{n} & a_{n} & \cdots & 0 & 0 \\
\cdots & \cdots & \cdots & \cdots & \cdots & \cdots \\
0 & 0 & 0 & \cdots & a_{n} & 0 \\
0 & 0 & 0 & \cdots & a_{n} & a_{n}
\end{array}\right]_{(N+1) \times(N+1)} \\
& B=\left[\begin{array}{cccccc}
b_{11} & 0 & 0 & \cdots & 0 & 0 \\
b_{21} & b_{22} & 0 & \cdots & 0 & 0 \\
b_{31} & b_{32} & b_{33} & \cdots & 0 & 0 \\
\cdots & \cdots & \cdots & \cdots & \cdots & \cdots \\
b_{N 1} & b_{N 2} & b_{N 3} & \cdots & b_{N N} & 0 \\
b_{N+1,1} & b_{N+1,2} & b_{N+1,3} & \cdots & b_{N+1, N} & b_{N+1, N+1}
\end{array}\right]_{(N+1) \times(N+1)} \\
& C=\left[\begin{array}{cccccc}
0 & 0 & 0 & \cdots & 0 & 0 \\
c_{n} & c_{n} & 0 & \cdots & 0 & 0 \\
0 & c_{n} & c_{n} & \cdots & 0 & 0 \\
\cdots & \cdots & \cdots & \cdots & \cdots & \cdots \\
0 & 0 & 0 & \cdots & c_{n} & 0 \\
0 & 0 & 0 & \cdots & c_{n} & c_{n}
\end{array}\right]_{(N+1) \times(N+1)} \\
& D=\left[\begin{array}{cccccc}
0 & 0 & 0 & \cdots & 0 & 0 \\
0 & 1 & 0 & \cdots & 0 & 0 \\
0 & 0 & 1 & \cdots & 0 & 0 \\
\cdots & \cdots & \cdots & \cdots & \cdots & \cdots \\
0 & 0 & 0 & \cdots & 1 & 0 \\
0 & 0 & 0 & \cdots & 0 & 1
\end{array}\right]_{(N+1) \times(N+1)} \\
& \varphi_{n}=\left[\begin{array}{c}
\varphi_{n}^{0} \\
\varphi_{n}^{1} \\
\varphi_{n}^{2} \\
\vdots \\
\varphi_{n}^{N-1} \\
\varphi_{n}^{N}
\end{array}\right]_{(N+1) \times 1}, \quad U_{q}=\left[\begin{array}{c}
u_{q}^{0} \\
u_{q}^{1} \\
u_{q}^{2} \\
\vdots \\
u_{q}^{N-1} \\
u_{q}^{N}
\end{array}\right]_{(N+1) \times 1} \quad, \quad q=n \pm 1, n, \\
& a_{n}=-\frac{1}{2}\left(\frac{1+x_{n}}{h^{2}}+\frac{1}{2 h}\right), \quad c_{n}=-\frac{1}{2}\left(\frac{1+x_{n}}{h^{2}}-\frac{1}{2 h}\right), \quad d=\frac{2}{\sqrt{\pi \tau}}, \\
& \lambda(r)=\sqrt{r+1 / 2}-\sqrt{r-1 / 2}, \quad \mu(r)=-\frac{1}{3}\left[(r+1 / 2)^{3 / 2}-(r-1 / 2)^{3 / 2}\right], \\
& b_{11}=1, \quad b_{21}=-\frac{d \sqrt{2}}{3}-\frac{1}{\tau}+\frac{1+x_{n}}{h^{2}}+\frac{1}{2}, \quad b_{22}=\frac{d \sqrt{2}}{3}+\frac{1}{\tau}+\frac{1+x_{n}}{h^{2}}+\frac{1}{2},
\end{aligned}
$$




$$
\begin{aligned}
& b_{31}=\frac{d 2 \sqrt{6}}{5}, \quad b_{32}=\frac{d \sqrt{6}}{5}-\frac{1}{\tau}+\frac{1+x_{n}}{h^{2}}+\frac{1}{2}, \quad b_{33}=\frac{d \sqrt{6}}{5}+\frac{1}{\tau}+\frac{1+x_{n}}{h^{2}}+\frac{1}{2}, \\
& b_{41}=d[\lambda(1)+\mu(1)], \quad b_{42}=d[-3 \lambda(1)-2 \mu(1)]-\frac{d}{6 \sqrt{2}}, \\
& b_{43}=d[2 \lambda(1)+\mu(1)]-4 \frac{d}{6 \sqrt{2}}-\frac{1}{\tau}+\frac{1+x_{n}}{h^{2}}+\frac{1}{2}, \quad b_{44}=5 \frac{d}{6 \sqrt{2}}+\frac{1}{\tau}+\frac{1+x_{n}}{h^{2}}+\frac{1}{2}, \\
& b_{51}=d[2 \lambda(2)+\mu(2)], \quad b_{52}=d[-5 \lambda(2)-2 \mu(2)+\lambda(1)+\mu(1)], \\
& b_{53}=d[3 \lambda(2)+\mu(2)-3 \lambda(1)-2 \mu(1)]-\frac{d}{6 \sqrt{2}}, \\
& b_{54}=d[2 \lambda(1)+\mu(1)]-4 \frac{d}{6 \sqrt{2}}-\frac{1}{\tau}+\frac{1+x_{n}}{h^{2}}+\frac{1}{2}, \quad b_{55}=5 \frac{d}{6 \sqrt{2}}+\frac{1}{\tau}+\frac{1+x_{n}}{h^{2}}+\frac{1}{2}, \\
& b_{i, j}= \begin{cases}d[(i-3) \lambda(i-3)+\mu(i-3)], & j=1, \\
d[(5-2 i) \lambda(i-3)-2 \mu(i-3)+(i-4) \lambda(i-4)+\mu(i-4)], & j=2, \\
d[(i-j+1) \lambda(i-j)+\mu(i-j)+(2 j-2 i+1) \lambda(i-j-1) & 3 \leq j \leq i-3, \\
-2 \mu(i-j-1)+(i-j-2) \lambda(i-j-2)+\mu(i-j-2)], & j=i-2, \\
d[3 \lambda(2)+\mu(2)-3 \lambda(1)-2 \mu(1)]-\frac{d}{6 \sqrt{2}}, & j=i-1, \\
d[2 \lambda(1)+\mu(1)]-4 \frac{d}{6 \sqrt{2}}-\frac{1}{\tau}+\frac{1+x_{n}}{h^{2}}+\frac{1}{2}, & j=i, \\
5 \frac{d}{6 \sqrt{2}}+\frac{1}{\tau}+\frac{1+x_{n}}{h^{2}}+\frac{1}{2}, & i<j \leq N+1 \\
0, & \end{cases}
\end{aligned}
$$

for $i=6,7, \ldots, N+1$ and

$$
\varphi_{n}^{k}=\left[3+k \tau+\frac{16 \sqrt{k \tau}}{5 \sqrt{\pi}}+(1+n h) \pi^{2} k \tau\right](k \tau)^{2} \cos (\pi n h)+\pi(k \tau)^{3} \sin (\pi n h) .
$$

For the solution of the matrix equation (4.2), we use the same algorithm as in the first order of accuracy difference scheme, where

$$
\begin{aligned}
& u_{M}=\left[3 I-4 \alpha_{M}+\alpha_{M-1} \alpha_{M}\right]^{-1} *\left[\left(4 I-\alpha_{M-1}\right) \beta_{M}-\beta_{M-1}\right] \\
& \alpha_{1}=E^{-1} F, \quad \beta_{1}=E^{-1} R \varphi_{0}, \\
& F=\left[\begin{array}{cccccc}
0 & 0 & 0 & \cdots & 0 & 0 \\
0 & 1 / h & 0 & \cdots & 0 & 0 \\
0 & 0 & 1 / h & \cdots & 0 & 0 \\
\cdots & \cdots & \cdots & \cdots & \cdots & \cdots \\
0 & 0 & 0 & \cdots & 1 / h & 0 \\
0 & 0 & 0 & \cdots & 0 & 1 / h
\end{array}\right]_{(N+1) \times(N+1)}, \\
& R=\left[\begin{array}{cccccc}
0 & 0 & 0 & \cdots & 0 & 0 \\
0 & 1 & 0 & \cdots & 0 & 0 \\
0 & 0 & 1 & \cdots & 0 & 0 \\
\cdots & \cdots & \cdots & \cdots & \cdots & \cdots \\
0 & 0 & 0 & \cdots & 1 & 0 \\
0 & 0 & 0 & \cdots & 0 & 1
\end{array}\right]_{(N+1) \times(N+1)}
\end{aligned}
$$




$$
\begin{aligned}
& E=\left[\begin{array}{cccccc}
e_{11} & 0 & 0 & \cdots & 0 & 0 \\
e_{21} & e_{22} & 0 & \cdots & 0 & 0 \\
e_{31} & e_{32} & e_{33} & \cdots & 0 & 0 \\
\cdots & \cdots & \cdots & \cdots & \cdots & \cdots \\
e_{N 1} & e_{N 2} & e_{N 3} & \cdots & e_{N N} & 0 \\
e_{N+1,1} & e_{N+1,2} & e_{N+1,3} & \cdots & e_{N+1, N} & e_{N+1, N+1}
\end{array}\right]_{(N+1) \times(N+1)} \\
& e_{11}=1, \quad e_{21}=-\frac{h}{4 \tau}-\frac{4 h}{3 \sqrt{\pi \tau}}, \quad e_{22}=\frac{1}{h}+\frac{h}{2}+\frac{4 h}{3 \sqrt{\pi \tau}}, \quad e_{23}=\frac{h}{4 \tau}, \\
& e_{31}=\frac{2 \sqrt{2} h}{15 \sqrt{\pi \tau}}, \quad e_{32}=\frac{-16 \sqrt{2} h}{15 \sqrt{\pi \tau}}-\frac{h}{4 \tau}, \quad e_{33}=\frac{1}{h}+\frac{h}{2}+\frac{14 \sqrt{2} h}{15 \sqrt{\pi \tau}}, \quad e_{34}=\frac{h}{4 \tau}, \\
& e_{41}=\frac{d h}{2}[(1+1 / 2) \lambda(1)+\mu(1)], \quad e_{42}=\frac{d h}{2}[-4 \lambda(1)-2 \mu(1)+1 / 2 \lambda(0)+\mu(0)], \\
& e_{43}=-\frac{h}{4 \tau}+\frac{d h}{2}[(2+1 / 2) \lambda(1)+\mu(1)-2-2(-1 / 3)] \text {, } \\
& e_{44}=\frac{1}{h}+\frac{h}{2}+\frac{d h}{2}[(1+1 / 2) \lambda(0)+\mu(0)], \quad e_{45}=\frac{h}{4 \tau}, \\
& e_{51}=\frac{d h}{2}[(2+1 / 2) \lambda(2)+\mu(2)] \text {, } \\
& e_{52}=\frac{d h}{2}[-2 \cdot 3 \lambda(2)-2 \mu(2)+(1+1 / 2) \lambda(1)+\mu(1)] \text {, } \\
& e_{53}=\frac{d h}{2}[(2+1+1 / 2) \lambda(2)+\mu(2)-2 \cdot 2 \lambda(1)-2 \mu(1)+1 / 2 \lambda(0)+\mu(0)], \\
& e_{54}=-\frac{h}{4 \tau}+\frac{d h}{2}[(1+1+1 / 2) \lambda(1)+\mu(1)-2 \lambda(0)-2 \mu(0)] \text {, } \\
& e_{55}=\frac{1}{h}+\frac{h}{2}+\frac{d h}{2}[(1+1 / 2) \lambda(0)+\mu(0)], \quad e_{56}=\frac{h}{4 \tau}, \\
& e_{i j}= \begin{cases}\frac{d h}{2}[(i-3+1 / 2) \lambda(i-3)+\mu(i-3)], & j=1, \\
\frac{d h}{2}[-2(i-2) \lambda(i-3)-2 \mu(i-3) & \\
\quad+(i-4+1 / 2) \lambda(i-4)+\mu(i-4)], & j=2, \\
\frac{d h}{2}[(i-j+1+1 / 2) \lambda(i-j)+\mu(i-j)-2(i-j) \lambda(i-j-1) & \\
\quad-2 \mu(i-j-1)+(i-j-2+1 / 2) \lambda(i-j-2)+\mu(i-j-2)], & 3 \leq j \leq i-2, \\
-\frac{h}{4 \tau}+\frac{d h}{2}[(2+1 / 2) \lambda(1)+\mu(1)-2 \lambda(0)-2 \mu(0)], & j=i-1, \\
\frac{1}{h}+\frac{h}{2}+\frac{d h}{2}[(1+1 / 2) \lambda(0)+\mu(0)], & j=i, \\
\frac{h}{4 \tau}, & j=i+1, \\
\frac{h}{4 \tau}+\frac{d h}{2}[(i-N+2+1 / 2) \lambda(i-N+1)+\mu(i-N+1) & \\
\quad-2(i-N+1) \lambda(i-N)-2 \mu(i-N) & \\
\quad+(i-N-1+1 / 2) \lambda(i-N-1)+\mu(i-N-1)], & j=N-1, \\
-\frac{h}{\tau}+\frac{d h}{2}[(2+1 / 2) \lambda(1)+\mu(1)-2 \lambda(0)-2 \mu(0)], & j=N, \\
\frac{1}{h}+\frac{h}{2}+\frac{3 h}{4 \tau}+\frac{d h}{2}[(1+1 / 2) \lambda(0)+\mu(0)], & j=N+1, \\
0, & j>i+1\end{cases}
\end{aligned}
$$

for $i=6,7, \ldots, N+1$ and

$$
\varphi_{0}^{k}=\left(3+k \tau+\frac{16 \sqrt{k \tau}}{5 \sqrt{\pi}}+\pi^{2} k \tau\right)(k \tau)^{2} .
$$


Table 1 Comparison of errors

\begin{tabular}{llll}
\hline Method & $\mathbf{N}=\boldsymbol{M}=\mathbf{3 0}$ & $\mathbf{N}=\boldsymbol{M}=\mathbf{6 0}$ & $\boldsymbol{N}=\boldsymbol{M = 1 2 0}$ \\
\hline 1st order difference scheme & 0.0910 & 0.0448 & 0.0222 \\
2nd order difference scheme & 0.0160 & 0.0040 & 0.0010 \\
\hline
\end{tabular}

\subsection{Error analysis}

Finally, we give the results of the numerical analysis. The error is computed by the following formula

$$
E_{M}^{N}=\max _{1 \leq k \leq N, 1 \leq n \leq M-1}\left|u\left(t_{k}, x_{n}\right)-u_{n}^{k}\right|,
$$

where $u\left(t_{k}, x_{n}\right)$ represents the exact solution and $u_{n}^{k}$ represents the numerical solutions of these difference schemes at $\left(t_{k}, x_{n}\right)$.

The numerical solutions are recorded for different values of $N$ and $M$. Table 1 is constructed for $N=M=30,60$ and 120 , respectively.

Thus, the results show that, by using the Crank-Nicholson difference scheme increases faster then the first order of accuracy difference scheme.

\section{Conclusion}

In the present study, the second order of accuracy difference scheme for the approximate solution of initial value problem (1.1) is presented. A theorem on almost coercivity of this difference scheme in maximum norm is established. Almost coercive stability estimates for the solution of the first and second order of accuracy stable difference schemes for the numerical solution of the initial boundary value problem for the fractional parabolic equation with the Neumann boundary condition are obtained. Of course, stability estimates permits us to obtain the convergence of difference schemes for the numerical solution of the initial boundary value problem for the fractional parabolic equation with the Neumann boundary condition. Moreover, the Banach fixed-point theorem and method of the present paper enables us to obtain the estimate of convergence of difference schemes of the first and second order of accuracy for approximate solutions of the initial-boundary value problem:

$$
\left\{\begin{array}{l}
\frac{\partial u(t, x)}{\partial t}+D_{t}^{\alpha} u(t, x)-\sum_{p=1}^{m} a_{p}(x) \frac{\partial^{2} u(t, x)}{\partial x_{p}^{2}}+\sigma u(t, x) \\
\quad=f\left(t, x ; u(t, x), u_{x_{1}}(t, x), \ldots, u_{x_{m}}(t, x)\right), \quad x=\left(x_{1}, \ldots, x_{m}\right) \in \Omega, 0<t<T, \\
\left.\frac{\partial u(t, x)}{\partial \vec{n}}\right|_{S}=0, \quad 0 \leq t \leq T, 0 \leq \alpha<1, \\
u(0, x)=0, \quad x \in \bar{\Omega}
\end{array}\right.
$$

for semilinear fractional parabolic partial differential equations with smooth $a_{p}(x)$ and $f\left(t, x ; u(t, x), v_{1}(t, x), \ldots, v_{m}(t, x)\right)$. 


\section{Author details}

'Department of Mathematics, Fatih University, Istanbul, Turkey. ${ }^{2}$ Department of Mathematics, ITTU, Ashgabat,

Turkmenistan. ${ }^{3}$ Department of Mathematical Engineering, Gumushane University, Gumushane, Turkey.

Received: 2 January 2013 Accepted: 11 April 2013 Published: 25 April 2013

\section{References}

1. Podlubny, I: Fractional Differential Equations. Mathematics in Science and Engineering, vol. 198. Academic Press, San Diego (1999)

2. Samko, SG, Kilbas, AA, Marichev, OI: Fractional Integrals and Derivatives. Gordon \& Breach, Yverdon (1993)

3. Kilbas, AA, Sristava, HM, Trujillo, JJ: Theory and Applications of Fractional Differential Equations. North-Holland Mathematics Studies. North-Holland, Amsterdam (2006)

4. Diethelm, K: The Analysis of Fractional Differential Equations. Lecture Notes in Mathematics, vol. 2004. Springer, Berlin (2010)

5. Diethelm, K, Ford, NJ: Multi-order fractional differential equations and their numerical solution. Appl. Math. Comput. 154(3), 621-640 (2004)

6. El-Mesiry, AEM, El-Sayed, AMA, El-Saka, HAA: Numerical methods for multi-term fractional (arbitrary) orders differential equations. Appl. Math. Comput. 160(3), 683-699 (2005)

7. Pedas, A, Tamme, E: On the convergence of spline collocation methods for solving fractional differential equations. J. Comput. Appl. Math. 235(12), 3502-3514 (2011)

8. El-Sayed, AMA, El-Mesiry, AEM, El-Saka, HAA: Numerical solution for multi-term fractional (arbitrary) orders differential equations. Comput. Appl. Math. 23(1), 33-54 (2004)

9. Gorenflo, R, Mainardi, F: Fractional calculus: integral and differential equations of fractional order. In: Carpinteri, A, Mainardi, F (eds.) Fractals and Fractional Calculus in Continuum Mechanics, vol. 378, pp. 223-276. Springer, Vienna (1997)

10. Yakar, A, Koksal, ME: Existence results for solutions of nonlinear fractional differential equations. Abstr. Appl. Anal. (2012). doi:10.1155/2012/267108

11. De la Sen, M: Positivity and stability of the solutions of Caputo fractional linear time-invariant systems of any order with internal point delays. Abstr. Appl. Anal. (2011). doi:10.1155/2011/161246

12. Chengjun, Y: Two positive solutions for $(n-1,1)$-type semipositone integral boundary value problems for coupled systems of nonlinear fractional differential equations. Commun. Nonlinear Sci. Numer. Simul. 17(2), $930-942$ (2012)

13. De la Sen, M, Agarwal, RP, Ibeas, A, Quesada, SA: On the existence of equilibrium points, boundedness, oscillating behavior and positivity of a SVEIRS epidemic model under constant and impulsive vaccination. Adv. Differ. Equ. (2011). doi:10.1155/2011/748608

14. De la Sen, M: About robust stability of Caputo linear fractional dynamic systems with time delays through fixed point theory. Fixed Point Theory Appl. (2011). doi:10.1155/2011/867932

15. Chengjun, Y: Multiple positive solutions for semipositone $(n, p)$-type boundary value problems of nonlinear fractional differential equations. Anal. Appl. 9(1), 97-112 (2011)

16. Chengjun, Y: Multiple positive solutions for $(n-1,1)$-type semipositone conjugate boundary value problems for coupled systems of nonlinear fractional differential equations. Electron. J. Qual. Theory Differ. Equ. 2011, 13 (2011)

17. Agarwal, RP, Belmekki, M, Benchohra, M: A survey on semilinear differential equations and inclusions involving Riemann-Liouville fractional derivative. Adv. Differ. Equ. (2009). doi:10.1155/2009/981728

18. Agarwal, RP, de Andrade, B, Cuevas, C: On type of periodicity and ergodicity to a class of fractional order differential equations. Adv. Differ. Equ. (2010). doi:10.1155/2010/179750

19. Agarwal, RP, de Andrade, B, Cuevas, C: Weighted pseudo-almost periodic solutions of a class of semilinear fractional differential equations. Nonlinear Anal., Real World Appl. 11, 3532-3554 (2010)

20. Berdyshev, AS, Cabada, A, Karimov, ET: On a non-local boundary problem for a parabolic-hyperbolic equation involving a Riemann-Liouville fractional differential operator. Nonlinear Anal. 75(6), 3268-3273 (2011)

21. Ashyralyev, A, Amanov, D: Initial-boundary value problem for fractional partial differential equations of higher order. Abstr. Appl. Anal. (2012). doi:10.1155/2012/973102

22. Ashyralyev, A, Sharifov, YA: Existence and uniqueness of solutions of the system of nonlinear fractional differential equations with nonlocal and integral boundary conditions. Abstr. Appl. Anal. (2012). doi:10.1155/2012/594802

23. Kirane, M, Laskri, Y: Nonexistence of global solutions to a hyperbolic equation with a space-time fractional damping. Appl. Math. Comput. 167(2), 1304-1310 (2005)

24. Kirane, M, Laskri, Y, Tatar, NE: Critical exponents of Fujita type for certain evolution equations and systems with spatio-temporal fractional derivatives. J. Math. Anal. Appl. 312(2), 488-501 (2005)

25. Kirane, M, Malik, SA: The profile of blowing-up solutions to a nonlinear system of fractional differential equations. Nonlinear Anal., Theory Methods Appl. 73(12), 3723-3736 (2010)

26. Araya, D, Lizama, C: Almost automorphic mild solutions to fractional differential equations. Nonlinear Anal. 69(11), 3692-3705 (2008)

27. N'Guerekata, GM: A Cauchy problem for some fractional abstract differential equation with nonlocal conditions Nonlinear Anal. 70, 1873-1876 (2009)

28. Mophou, GM, N'Guerekata, GM: Mild solutions for semilinear fractional differential equations. Electron. J. Differ. Equ. 2009, 21 (2009)

29. Mophou, GM, N'Guerekata, GM: Existence of mild solution for some fractional differential equations with nonlocal conditions. Semigroup Forum 79(2), 315-322 (2009)

30. Lakshmikantham, V: Theory of fractional differential equations. Nonlinear Anal. 69(10), 3337-3343 (2008)

31. Lakshmikantham, V, Devi, JV: Theory of fractional differential equations in Banach spaces. Eur. J. Pure Appl. Math. 1, 38-45 (2008)

32. Ashyralyev, A, Dal, F, Pınar, Z: A note on the fractional hyperbolic differential and difference equations. Appl. Math. Comput. 217(9), 4654-4664 (2011)

33. Ashyralyev, A, Hicdurmaz, B: A note on the fractional Schrödinger differential equations. Kybernetes 40(5/6), 736-750 (2011) 
34. Cakir, Z: Stability of difference schemes for fractional parabolic PDE with the Dirichlet-Neumann conditions. Abstr. Appl. Anal. (2012). doi:10.1155/2012/463746

35. Ashyralyev, A, Cakir, Z: On the numerical solution of fractional parabolic partial differential equations with the Dirichlet condition. Discrete Dyn. Nat. Soc. (2012). doi:10.1155/2012/696179

36. Clement, P, Delabrire, SG: On the regularity of abstract Cauchy problems and boundary value problems. Atti Accad. Naz. Lincei Cl. Sci. Fis. Mat. Natur. Rend. Lincei Mat. Appl. 9(4), 245-266 (1999)

37. Agarwal, RP, Bohner, M, Shakhmurov, VB: Maximal regular boundary value problems in Banach-valued weighted space. Bound. Value Probl. 1, 9-42 (2005)

38. Lunardi, A: Analytic Semigroups and Optimal Regularity in Parabolic Problems. Operator Theory: Advances and Applications. Birkhäuser, Basel (1995)

39. Ashyralyev, A, Hanalyev, A, Sobolevskii, PE: Coercive solvability of nonlocal boundary value problem for parabolic equations. Abstr. Appl. Anal. 6(1), 53-61 (2001)

40. Ashyralyev, A, Hanalyev, A: Coercive solvability of parabolic differential equations with dependent operators. TWMS J. Appl. Eng. Math. 2(1), 75-93 (2012)

41. Ashyralyev, A, Sobolevskii, PE: Well-Posedness of Parabolic Difference Equations. Birkhäuser, Basel (1994)

42. Ashyralyev, A: Well-posedness of the Basset problem in spaces of smooth functions. Appl. Math. Lett. 14(7), 1176-1180 (2011)

43. Basset, AB: On the descent of a sphere in a viscous liquid. Q. J. Math. 42, 369-381 (1910)

44. Ashyralyev, A: Well-posedness of parabolic differential and difference equations with the fractional differential operator. Malays. J. Math. Sci. 6(S), 73-89 (2012)

45. Ashyralyev, A: A note on fractional derivatives and fractional powers of operators. J. Math. Anal. Appl. 357(1), 232-236 (2009)

46. Ashyralyev, A, Sobolevskii, PE: Theory of the interpolation of the linear operators and the stability of the difference schemes. Dokl. Akad. Nauk SSSR 275(6), 1289-1291 (1984)

47. Alibekov, HA, Sobolevskii, PE: Stability and convergence of difference schemes of a high order of approximation for parabolic partial differential equations. Ukr. Mat. Zh. 32(3), 291-300 (1980)

48. Alibekov, HA, Sobolevskii, PE: The stability of difference schemes for parabolic equations. Dokl. Akad. Nauk SSSR 232(4), 737-740 (1977)

49. Sobolevskii, PE: The coercive solvability of difference equations. Dokl. Akad. Nauk SSSR 201(5), 1063-1066 (1971)

50. Samarskii, AA, Nikolaev, ES: Numerical Methods for Grid Equations: Iterative Methods, vol. 2. Birkhäuser, Basel (1989)

doi:10.1186/1687-1847-2013-120

Cite this article as: Ashyralyev and Cakir: FDM for fractional parabolic equations with the Neumann condition. Advances in Difference Equations 2013 2013:120

\section{Submit your manuscript to a SpringerOpen ${ }^{\ominus}$ journal and benefit from:}

- Convenient online submission

- Rigorous peer review

- Immediate publication on acceptance

- Open access: articles freely available online

High visibility within the field

- Retaining the copyright to your article 